\title{
One Ring to Unite Them All: Convergence, the Smartphone, and the Cloud
}

\author{
Bryan Pon • Timo Seppälä • Martin Kenney
}

Received: 4 April 2014 /Revised: 12 July 2014/

Accepted: 3 December 2014 /Published online: 13 February 2015

(C) The Author(s) 2015. This article is published with open access at Springerlink.com

\begin{abstract}
This paper examines how recent trends in the smartphone industry may be expanding previous conceptions of the industry and its boundaries. The increasing importance of Internet and cloud-based services - which in many ways lie outside the control of the physical device, operating system, and even the cellular network - seems to be changing the roles and strategies of key firms in the ecosystem. Using industry architecture and platform theory, we examine how the key firms seem to be reacting to these new changes. Our analysis indicates that the platform "bottleneck," or key control point, is moving away from the device and into the cloud, where a new meta-platform based on the Internet may be emerging.
\end{abstract}

Keywords Smartphone $\cdot$ Platform $\cdot$ Bottleneck $\cdot$ Apple iOS · Android

JEL Classification $\mathrm{L} 14 \cdot \mathrm{L} 15 \cdot \mathrm{L} 86 \cdot \mathrm{L} 96$

\section{Introduction}

Convergences are particularly interesting industrial periods because at these moments entire industries can be restructured as firms compete to achieve positions of architectural advantage. Today, the long discussed digital convergence appears to be finally occurring, with telecommunications in the form of the Internet and cloud computing at its unifying center, but with a myriad of intelligent communicating and sensing devices at its edges. In the still-evolving smartphone space, firms from the telephony, computing, and internet industries have come into competition with each other and are attempting to navigate the convergence using a range of

B. Pon $(\bowtie) \cdot$ M. Kenney

Community and Regional Development Unit, Geography Graduate Group, University of California, 1

Shields Ave., Davis, CA 95616, USA

e-mail: bjpon@ucdavis.edu

M. Kenney

e-mail: mfkenney@ucdavis.edu

T. Seppälä

Department of Industrial Engineering and Management, Aalto University \& ETLA, The Research Institute of the Finnish Economy, Lönnrotinkatu 4B, Helsinki 00120, Finland

e-mail: timo.seppala@etla.fi 
platform strategies. Those strategies are situated within an increasingly complex technological and economic space, characterized by multi-sided markets, a significant variety of business and revenue models, ever-advancing technologies, changing standards and regulations, and the layered nature of the computer-telephony industry itself.

Theory suggests that in the information and communication technology (ICT) industries, technology platforms are a key way in which firms can control value creation and position themselves to capture a disproportionate share of industry profits (Gawer 2011; Jacobides et al. 2006). By developing a core platform and attracting complementors to build on and extend it, platform firms harness external innovation to create value for the entire ecosystem. Successful platforms are able to attract both complementors and end-users in a virtuous cycle of network effects, which can lead to dominant market share and entrenched competitive advantage for the firm controlling the platform (Katz and Shapiro 1985). Kenney and Pon (2011) considered a number of the key players in terms of platform theory, and found that Google and Apple's smartphone strategies were at odds with the traditional platform theory as described by Gawer and Cusumano (2002). This paper extends that analysis beyond the device and the operating system to focus on the role of Internet-based services, and how they may be influencing firms' platform strategies (for a more general discussion of platforms and services, see Cusumano and Suarez 2009).

This paper has two goals: first, to demonstrate that the increasing importance of the Internet and cloud-based services is moving platform power from the device into the cloud. If true, this shift has significant implications for firm strategy, as it signifies not only a new playing field where the competition will be waged, but an opportunity for restructuring power and relationships within the merging value networks. One key trend is the convergence in smartphone hardware and software, which suggests that a dominant design, based on the iPhone, has been established. Beyond the smartphone itself, there is a proliferation of other "smart" and "sensing" devices connected to the Internet—from mobile devices such as tablets, watches, and automobiles, to stationary devices such as TVs, refrigerators, and home energy thermostats - illustrating the broad range of functionality that is almost certain to be connected wirelessly. Even as this intelligent hardware diversifies and multiplies, the long-running trend toward data processing and storage in the cloud is accelerating. Software-as-a-service (SaaS) appears to be the future for not only mobile devices, but most devices including the desktop computer. The result of these trends looks to be a quality and degree of connectedness that only a decade ago was unimaginable.

The second goal is to explain how the key platform firms are responding to these shifts in the mobile industry landscape. We apply concepts from the industry architecture and platform literatures to interpret the actions of Apple, Google, and Microsoft, who have all launched mobile operating systems that have become powerful and, for some, profitable technology platforms. The platform literature suggests that the key to securing architectural advantage is owning the "bottleneck," or the core asset that is used across complementors within the ecosystem, yet has high barriers to entry. This allows the owner to extract rents from those requiring the platform for their businesses (Jacobides et al. 2006). Up to this point, similar to the PC industry, the bottleneck in the smartphone industry has been the operating system installed on the device. However, the increasing centrality of cloud services suggests that the locus of power may be shifting from the operating system to the service providers. For the platform firms, this forces strategic decisions around the scope of their business, and whether they need to strengthen or fully integrate complementary assets to maintain control over a shifting bottleneck. Given the substantially distinct business models of Apple, Google, and Microsoft, this analysis raises questions around the traditional tradeoffs between vertical and horizontal integration - a dichotomy that may not mean much in complex, multilayered 
techno-economic systems. This complexity is underscored by the emergence of new platforms from Amazon and Xiaomi, who have each built proprietary platforms on top of Google's opensource Android OS (Pon et al. 2014). We conclude with a discussion of how this shift in mobile telephony is part of broader alignment of many types of networked devices and service platforms connecting to the cloud through Internet protocols, thereby creating a "metaplatform" or additional layer that may challenge firms' platform strategies.

\section{From the Device to the Cloud}

Demand for smartphone handsets has seen explosive growth in the last decade, enticing a wide range of manufacturers and complementors to enter the market. Yet even as the competitive landscape intensifies, handsets show signs of convergence in functional specifications and physical design. The flagship handsets of the major handset manufacturers-Samsung, LG, HTC, Xiaomi, Nokia, and even Blackberry - score similarly on performance benchmarks, and have, for the most part, emulated the design defined by the original Apple iPhone (West and Mace 2010). This increasing similarity suggests that a "dominant design" for smartphones has emerged.

The concept of a dominant design has been explored in the industrial organization literature (e.g., Anderson and Tushman 1990), technology and innovation literature (e.g., Abernathy and Utterback 1978; Teece 1986), and economics literature around standardization (e.g., Farrell and Saloner 1992; Katz and Shapiro 1985). Dominant design theory characterizes product innovation as cyclical, such that when a radical, "discontinuous" innovation is introduced, firms rapidly iterate and experiment until a dominant design is established, after which innovation is incremental (Anderson and Tushman 1990). Once a dominant design is established, it is unlikely to be challenged until the next discontinuous innovation, especially in industries with high barriers and costs to prototyping and manufacturing - what Teece (1986) calls "significant irreversibilities." Work by Abernathy and Utterback (1978) suggests that the emergence of a dominant design will shift firm strategies: prior to the emergence of the dominant design, resources and innovation are product-based as firms race to discover the optimal design; once that design is established, the innovative effort shifts toward processbased improvements bringing down costs and improving operational and service efficiencies.

Much of this is due to requirements of the technology itself - in the experimentation phase, firms are developing new product combinations with cutting-edge technologies that may have not been used together, so the degree of modularity and codified knowledge will be low, requiring a more integrated approach to design and manufacturing. As the dominant design becomes widespread, components generally become more modular and commoditized with more standardized interfaces. This transition prompts firms to move from vertical integration toward modular approaches (Baldwin and Clark 2000; Christensen and Anthony 2004). The high level of standardization and modular design allow individual firms to specialize and reach economies of scale in their area of specialization.

While the emergence of a dominant design thus typically includes processes of standardization, these phenomenon are distinct in that any given product design may be comprised of many different standards, some of which may be developed through de facto market processes (e.g., Webkit engine for mobile browsers) while others may come through formal de jure processes (e.g., micro-USB ports). Furthermore, a dominant design may include technical components that appear standardized to users, but can be implemented with a wide range of underlying technologies (e.g., the glass touchscreen is an essential component of the smartphone dominant design, yet there are dozens of different technologies that can be used 
to implement this feature). The characteristics that drive adoption of standards - economies of scale, interoperability, and network effects - therefore apply to a dominant design at different scales depending on the unit of analysis.

However, the clear emergence of a dominant design for the smartphone and its user interface has not yet been accompanied by a shift toward modularity. Apple, which has always been tightly integrated, has expanded its integration of previously complementary assets, including customizing the design of its mobile SoC (system on a chip) starting with the A6. Google purchased and then sold Motorola Mobility, and while it stated that it had no intention of operating as an integrated software-hardware unit, Google has also built a laptop. Perhaps most tellingly, Microsoft - the quintessential horizontal firm-in 2012 declared itself a "devices and services company," and built its own hardware with the Surface tablet. This move into hardware, including the purchase of Nokia's handset operations, threatens important members of Microsoft's large ecosystem of hardware systems providers. Put differently, the increasing importance of the smartphone and other portable devices appears to be forcing Microsoft to attack loyal complementors of its Windows ecosystem. These strategic moves exploring integration (or, in the case of Google integration and then retreat) suggest that either a dominant design is not yet in place, or that the industrial boundaries within which integration is understood need to be broadened. For example, one explanation is that while the mobile handset has reached a plateau, firms are looking ahead to the potential variety of other mobile devices that may supplement, or displace, the traditional handset (e.g., smartwatches; for a discussion see Seppälä and Broens 2013). This suggests that key competitors do not yet understand the ultimate configuration of the industry.

\subsection{Operating Systems and Software}

Whether the convergence in design and functionality extends to the operating system and key software is less clear. Up to this point, the operating system (OS) has been arguably the key differentiator between smartphones and respective ecosystems, retracing the path of the personal computer industry (i.e., Windows vs. Apple II vs. C/PM); a struggle that provided the conceptual underpinnings for platform theory. Understanding the future through memories of the past is common. For this reason, the OS has been the key for the platform battle among firms who see platform market share as essential to industry success.

In 2013, the smartphone market is dominated by two operating systems, with Android and iOS shipping on $78 \%$ and $16 \%$ respectively of all smartphones sold worldwide. Other firms - especially Windows Phone and BlackBerry, but including Firefox, Tizen, Sailfish, and Ubuntu - are promoting their own OS in an effort to make inroads against the reigning Android/iOS duopoly, but with limited success. While the iPhone disrupted the market dominance of Symbian and other incumbents 7 years ago, the unprecedented rate of adoption for Android — on track to be the most quickly adopted technology platform in history - is again reconfiguring the balance of power (Fig. 1).

There are other signs of increasing cross-platform functionality in the industry. Jolla's Sailfish operating system is designed to run slightly modified Android apps in addition to native Sailfish apps, giving the new platform a jumpstart in the app arms race. For developers, the practice of "multi-homing," or developing apps for multiple platforms, is common, and new software tools make it easier than ever to port successful apps from one platform to another. However, for the smaller platforms, the much lower number of apps (in early 2014, Windows Phone had 240 000, vs. over 1 million for both iOS and Android) are negatively affecting adoption of those alternatives. 


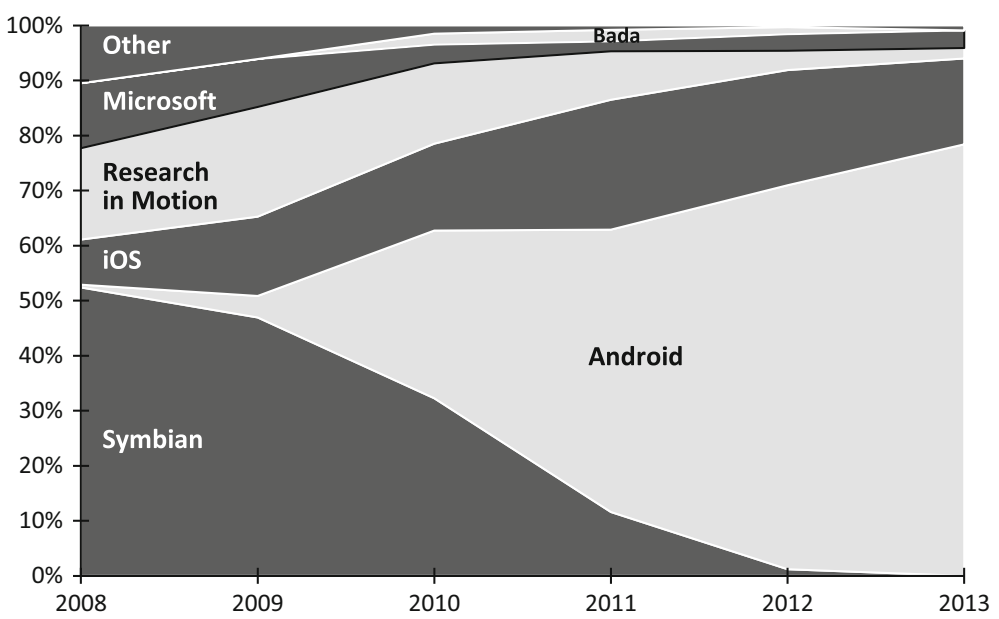

Fig. 1 Global market share of smartphone operating systems. Source: Gartner reports

\subsection{Served from the Cloud}

Software has been moving from the desktop to the cloud for decades. For many consumer and enterprise applications, software is now hosted in the cloud and runs in the browser, so that the user does not have to download or install anything locally. Early success stories such as Salesforce.com established software-as-a-service (SaaS) or on-demand software as a preferable alternative to expensive and difficult to service enterprise software such as Oracle or SAP. The trend is gaining momentum, fueled by new software and the decreasing costs of data storage, processing power, and bandwidth, such that even the most valuable software suite of all time, Microsoft Office, is offering cloud-based versions to compete with Google Docs, Zoho, and other online productivity software. For end-users, what started with cloud-based email has now grown to cloud-based calendars, music, photos, file storage, contacts, and more. Increasingly, data of all types is being stored on the server, where it can be accessed from a multiplicity of edge devices and mobile apps. While smartphones originally imitated the desktop model and accessed web sites and services such as Gmail through the browser, the explosion in dedicated mobile apps for Internet services has mostly supplanted web use-instead of going to Google.com, Netflix.com, or Facebook.com, users increasingly download the Google, Netflix, and Facebook apps. Importantly, this shift has ramifications for the platform, as users must typically go through a dedicated and restricted online marketplace (e.g., Apple App Store) for the apps, while browsing directly to the website is unrestricted by the platform owner. In sum, the services that enable users to communicate, navigate, be productive, consume media, and play games are increasingly based in the cloud, making a network connection a prerequisite for most mobile computing activity.

\subsection{Networked Devices}

While there is evidence of a trend toward convergence in smartphones per se, there is also clearly expansion in other types of mobile and sensing devices or contexts in which mobile operating systems and technology are being used. The adoption of tablets is growing at a tremendous rate, and many analysts estimate that total shipments will surpass PCs in 2015. 
But mobile operating system and wireless technology functionality is also being embedded in TVs, home energy systems, refrigerators, automobiles, watches, glasses, and more. As the cost of key components drops and technology miniaturization continues, the number of connected devices is multiplying, finally beginning to fulfill the vision of the "Internet of Things" (Chui et al. 2010; Gerschenfeld et al. 2004). As more types of devices become connected to the Internet, the ability to manage these devices and transform the data being transmitted into valuable information becomes an enticing financial opportunity. Besides the obvious stakes in the smartphone industry, success in smartphones may be extendable to the Internet of Things, where the smartphone could play a central role.

\section{Platforms and Bottlenecks}

For firms in emerging new industries or those being transformed by new technologies, identifying the key positions for capturing outsized shares of the value generated is critical. Recent understanding of firm strategic positioning has been based on analysis of how ICT leaders such as Microsoft, Intel, Cisco, Qualcomm and others have achieved sustained profits by controlling a key technology - the "platform" upon which "other firms can develop complementary products, technologies, or services" (Cusumano 2010; Cusumano and Gawer 2001; Gawer 2011). Platforms benefit from direct and indirect network effects, or "network externalities," whereby the value of being on the platform increases with the number of users of the platform (Katz and Shapiro 1985; Rochet and Tirole 2003). When a platform reaches a critical mass of users, these network effects operate as a de facto barrier to entry, which helps explains how Microsoft Windows has been able to stay dominant in the PC industry for decades (Tee and Gawer 2009). Although platform owners or "sponsors" may control specific IP or assets, they are dependent on the ecosystem of complementors to add value to the platform, and therefore need to develop processes and structures that incentivize complementors to innovate (Schilling 2009; West 2003; Gawer 2011).

\subsection{Bottlenecks in the Cloud}

While the concept of a platform is useful in understanding how the ecosystem of platform owners and complementors negotiate their relationships, we extend the platform model to include the idea of technology "bottlenecks." As described by Jacobides et al. (2006), bottlenecks are those focal points where "de facto exclusion of possible producers limits entry into particular segments of the industry architecture, whereas mobility (both in terms of switching costs and potential entry) is high in others." Operating systems can be natural bottlenecks, as there are typically high switching costs (developing hardware and software complements for one OS vs. another) and high barriers to entry (witness Samsung and Nokia's lack of success thus far in introducing their own mobile operating systems). The iconic example is the personal computing industry, where the operating system or closely tied native applications (e.g., Microsoft Windows and Office) have been the platform bottleneck and locus for value capture (and were the subject of significant anti-trust litigation; see Klein 2001). For decades the PC has been used as a hardware device with value-added products - software - as complementary goods necessary for users to conduct computing tasks. Although the Internet began shifting the role of the PC, as Cusumano and Yoffie (1998) show, Microsoft was able to leverage its ownership of the PC operating system to rebuff Netscape and ensure that the PC industry structure and OS bottleneck remained unshaken. This model of operating-system-as- 
bottleneck is being challenged in the mobile industry, most directly by the emergence of new proprietary platforms from Amazon and Xiaomi built on top of the open-source Android OS. That these new platforms - complete with walled garden app stores and integrated servicesshare the same operating system foundation provides further evidence that the OS is no longer the most important control point (Pon et al. 2014).

Mobile phones are predicated upon connectivity, and as network speeds and computing power have increased to allow ever more functionality via the Internet, a new industrial landscape is emerging with devices being integrated into the cloud services to which they connect. The contrast is important. For PC users, the value was in the device and its application software (of which the Office suite and a few others, such as Adobe Acrobat, were primary), allowing Microsoft and Intel to lock in users and establish control points in their layers in the stack (see Kenney and Curry 2002). For smartphone users, the value is in the content and services that the device connects to, forcing smartphone firms to look beyond the device for complementary assets and lock-ins.

The services, apps, and media that users want are typically accessed through online digital marketplaces and user accounts (though the app may be free). These accounts include personal information as well as credit card or payment processing information, and are required for using the App Store, Google Play, or Windows Phone Store. For Apple's and Microsoft's platforms, if a user wants to download an app, purchase a movie, or find a podcast, they must do so through their user account in the platform's online marketplace. This tightly curated walled garden provides greater control over complementary goods and a defined way of capturing value. Both Apple and Microsoft have extended this model and are using similar online marketplaces - the Mac App Store and Windows Store - as the central source for desktop software as well.

While Google's platform offers greater flexibility in accessing and downloading contentusers can download and install apps from 3rd-party sources such as Amazon, Xiaomi, SlideME, and GetJar - Google Play is the official marketplace for Android apps, with the highest number of apps and users. Payment on Google Play is also more flexible - even though Google has a fully developed payment processing service, Google Wallet, it has arranged contracts with some network operators for pass-through billing for Google Play purchases. Here again, Google's strategy departs from the more traditional platforms of Apple, Microsoft, and BlackBerry, in that it allows users of its platform to access content and apps from other sources; Google has built a garden, but there is no wall around it (Fig. 2).

\subsection{Platform Interoperability}

This model of integrated cloud services is employed across the leading smartphone firms: Apple, Google, and Microsoft all have online marketplaces with millions of user accounts that not only allow the user to easily purchase digital goods, but also link to the user's cloud-based email, file storage, calendar/contacts, and more. These services have varying degrees of adoption, and many users mix services from different platforms. For example, many users prefer Gmail for their primary email account, but use an iPhone device. These scenarios are increasingly common, and bring up important strategic questions about interoperability. Previous research has shown that platforms that become dominant tend to avoid interoperability with weaker platform rivals, or may provide some interoperability but on a lower level of service in order to maintain competitive differentiation (Eisenmann et al. 2009). These assumptions, however, presume that different platform owners are competing along the same dimensions. And yet, due to divergent revenue models among the top smartphone platform owners - e.g., product sales, OS licensing, advertising - the vectors of competition are 


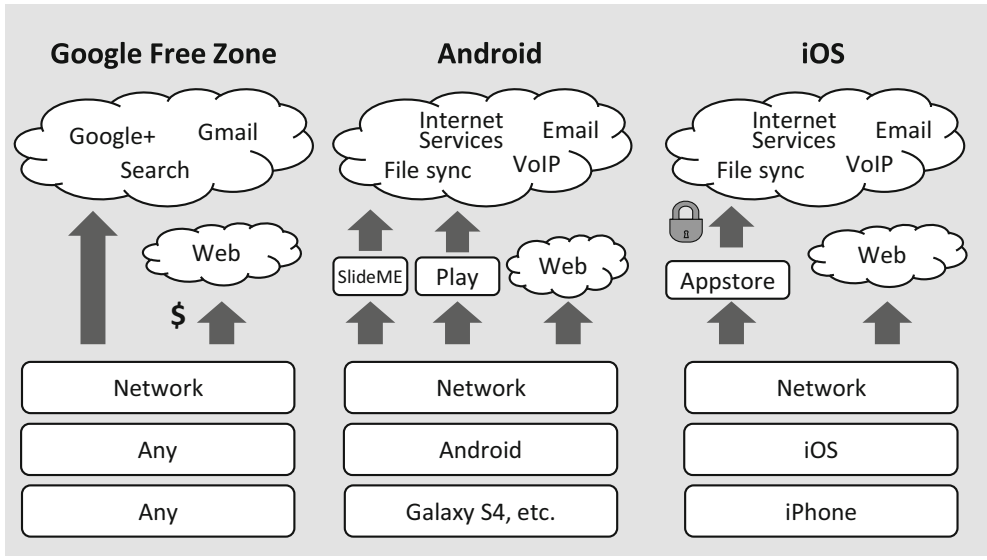

Fig. 2 Controlling access through online marketplaces. Notes: On right, Apple's walled garden approach restricts access to internet services - user must go through the platform marketplace, creating a bottleneck of control. The same approach is used by Microsoft, BlackBerry, and Amazon. The more open model of Google (center) allows the user to access Internet services through any number of 3rd-party marketplaces, such as SlideME, as well as the official Google Play market. In both cases, most Internet services are available to either platform due to increasing interoperability (e.g., Gmail on iPhone). Access to the web is unrestricted for both models. In emerging economies of Africa and Southeast Asia, the Google Free Zone and Facebook Zero model (left) works on any web-enabled device and any operating system, incentivizing low-income users to access a "pay-walled garden" of free services offered by Google or Facebook. Access to the web beyond Google or Facebook is unrestricted, but requires purchase of a data plan

complex and not always aligned, making it difficult to anticipate the appropriate strategy for interoperability.

Among the main smartphone platforms, most of the major cloud services have apps or APIs to support their function on a different platform's device, but there have been clashes. For example, when Apple launched the iPhone 5, it attempted to replace Google Maps with its own native map app, but quickly failed and retreated. For Apple, the decision to offer a Google alternative may have been driven by the fact that Google Maps functionality updates were slower for iOS vs. for Android. What Apple discovered was that what appeared as a simple substitution, in fact, was a consumer-demanded lock-in. For traditional platform owners, decisions to interconnect with other platforms and services depend in large part on the relative strength of the platforms involved, the potential increase in user base or market size, and considerations of competitive differentiation (Boudreau 2010; Eisenmann et al. 2011).

Given the success of Google's "open" Android offering, it is harder to predict how it will use its other complementary assets. When Apple retreated from its replacement of Google Maps, it had to ask Google to introduce a new app for iPhone users to install. By agreeing to do so, Google illustrated the importance of Internet services to users. Effectively, Google (the service provider) affects the device users' experience. To illustrate, when the Google Now service was originally introduced, it was only available on the Android platform, and was not available for iOS users until months later during which time it served as a competitive differentiator for Android. A thought experiment from the user perspective may help us to understand where the power lies; i.e., where the industry bottleneck is located: If Google stopped making its services such as Maps, YouTube, and Gmail compatible with other platforms, how many users would prefer to give up their Google services, compared to how many would prefer to switch to a different phone? 


\subsection{Services Take Over}

The role of the operating system is also being threatened by services taking on a larger presence on the device itself. The Facebook Home application for Android, which largely failed, was meant to replace the normal OS home screen and lock screen by showing real-time feeds and posts from the user's Facebook account. By allowing the user to interact directly with the Facebook service without launching an app or browser, Facebook Home was an attempt to blur the boundaries between app and operating system for the average user. For Facebook, the Home application put the Facebook service front and center, making it even easier for users to engage with their social network. For Google, as the platform owner, this displacement of the standard Android menus and controls does not directly threaten its services, since Facebook does not directly compete with Google's core services of search, maps, video, etc.

Facebook has developed other ways to extend its service into the core functionality of mobile devices. Beginning in 2010, Facebook rolled out Facebook Zero, a "zero-rating" service that provides Facebook content for free to mobile users. Targeting regions such as sub-Saharan Africa and Southeast Asia, Facebook Zero offers users the ability to read and reply to Facebook posts using a feature phone-for free, without a data plan. The network operators cover the costs for the access, and only charge the user for data if the user tries to download photos or access web content outside of the Facebook site. For Facebook, the service is part of its strategy for increasing its user base in the emerging markets, where subscription growth rates far outpace the more saturated markets of North America and Europe. For operators, the free access to a popular website familiarizes users with connecting to the Internet, and may lead some users to start paying for data plans, bringing them into the smartphone market. In response, Google in 2012 launched its Free Zone service, which also works with network operators to provide free internet access to a selected group of sites: Gmail, Search, and Google+. Both Facebook Zero and Google Free Zone are able to run on web-enabled feature phones, and as Internet-based services, they are not tied to any operating system. By offering a clear financial incentive for low-income users-free, if very limited, Internet access-Facebook and Google are bypassing the operating system with a direct connection to their respective Internet services. Even for those users who may have an Android or BlackBerry device, the free access could be more compelling than purchasing a data plan in order to browse the general web or go through the associated platform's online marketplace for apps and other content. Therefore for many of these users, the service is their only experience of the Internet.

\subsection{The Internet as a Meta-Platform}

Beginning in the decade of 2000, the Internet and its "cloud" manifestation have ignited fundamental reorganization of the ICT world. Technological changes are creating an "economics of abundance" in processing power, data storage, and network bandwidth, leading to new use cases and business models for both mobile computing and cloud-based services (Murray 2013). The phone is becoming a mobile computing device rather than a telephone, the PC is becoming a powerful tablet for many users, and the TV is becoming "smart" with its own computing functions. While these three defining networked devices are experiencing significant increases in functionality, the perhaps more important change is in the nature of network communication.

In the previous telecommunications regime, the telephone (wired and wireless), television, and personal computer all connected to the digitized central information delivery backbone via 
networks that were not fully compatible. Driven by the technological abundance in processing power and changes in user demand for Internet services, the phone, and later the TV, became sufficiently "smart" with processing and communication power to enable new applications and functionality. The smartphone is now increasingly a device for connection to Internet services rather than for direct verbal communication with other users (the use of services such as Skype routes verbal communication through the Internet). The TV also has its own processors and communications chips inside, enabling it to service the user demand for an expanding range of non-network television content. As a result, all three devices are increasingly connected to the Internet, and thus communicate via the same TCP/IP and related protocols. Moreover, the "Big Three" are being joined by yet other computing and communication capability-endowed devices, such as automobiles, refrigerators, personal health monitors, cameras, and a plethora of other electronic gadgets. When, not if, these devices become connected to the Internet, then the heart of the convergence will be the cloud data center, where the data going to and coming from the range of different devices would be served from and stored at (Kushida et al. 2011). The Internet would be the core platform to which all others connect - the meta-platform for ICT.

This abundance has not only transformed technology at the device level, but has created and likely will continue to create entire new sectors and business models around cloud-based services. In contrast to devices, which have become smaller and smaller, the growth in cloud services is about getting bigger and bigger: larger data centers, more servers and databases, and higher network bandwidth. An entire new class of service providers is now providing "infrastructure-as-a-service" (IaaS, e.g., servers and databases) and "platform-as-a-service" (PaaS, e.g., software installed on servers) to allow firms to quickly and flexibly build, test, and deploy services using a ready-made, scalable infrastructure and programming libraries. Strikingly, three of the market-leading cloud-based operators are Google (App Engine), Microsoft (Azure), and Amazon (AWS). Perhaps equally interesting, neither Apple nor Samsung have significant assets in this space. How, or whether, these cloud-based development environments can contribute to the firms' mobile platforms is unknown, but the fact that neither Apple nor Samsung have significant assets in this space shows clear strategy differences. The business case for these "factories of the future" is based in economies of scale, with cheap processing, memory, and bandwidth enabling the collection, service, and monetization of ever-increasing amounts of data. Yet it may be the firms finding innovative ways to apply economies of scope to their cloud computing centers will be the ones able to leverage these assets strategically in the Internet platform battle.

\section{Conclusion}

Technological convergences not only merge previously separate industries, but can also force previously distinct roles in the value chain to redefine their relationships to other actors. In the smartphone industry, the convergence of telephony, computing, and the Internet has led to new structures of competition among firms promoting different technology platforms and business models. It is important to note that at the moment, the different business models mean that this competition is asymmetric, making outcomes particularly difficult to predict.

What is increasingly apparent is that the battleground of competition is shifting power away from the device and operating system as platforms, and toward Internet-based services linked to user accounts and data. At the handset level, there is clear convergence in hardware design and function with the dominant design largely based upon the original iPhone. On top of the handset, mobile operating systems and apps are showing increased compatibility and cross- 
platform availability, driven in part by the rise in popularity of open standards and cloud services. Users are increasingly turning to Internet-based services for not only email and calendars, but for streaming media, file storage, video conferencing, real-time collaboration, and more, such that what matters for users is not the operating system on the device, but what the device allows them to connect to. This shift toward the cloud moves the critical juncture, the "bottleneck," away from the device itself and to the services available in the cloud. For the smartphone, all of the platforms currently regulate, in some way, how devices can connect to these services, typically through an online marketplace with varying degrees of open-ness. Controlling this environment as a gateway to the services that users care about is critical, and may be more important than controlling the operating system itself. This disintermediation of the operating system from its ecosystem of cloud services - explicitly allowed by Google's Android Open Source Project - is enabling service providers such as Xiaomi and Amazon to create and capture value with Android as if they were the platform owner (Pon et al. 2014). Google is a nontraditional platform owner due to its monetization through advertising, but direct threats to its ability to capture value from Android users do not yet seem to have materialized - largely because of the value of the services it provides. Further, in the same way, as in the PC world, Microsoft benefitted from selling the Office suite for Apple Macintosh computers, Google benefits with every smartphone sold - if the user utilizes Google services.

For the platform owners, these shifts pose strategic challenges, and they must determine which complementary assets will be key to growth. To date, vertical integration has helped Apple and Samsung capture the majority of the profits in the industry by selling devices (in the latter case, also components). For these hardware-centric firms, there is price pressure. Lowercost devices from Xiaomi, ZTE, and Huawei, for example, are becoming more sophisticated yet are priced at a fraction of the cost of premium handsets. Google and Microsoft, which have more substantial Internet services compared to Apple, BlackBerry, or Samsung, may be better positioned to integrate their Internet offerings into their products and services and lock-in users. With their IaaS/PaaS offerings, both companies may be able to leverage their storage and processing resources to improve their mobile platforms in innovative ways - for example, Microsoft could offer developers a specialized service or API for its Azure PaaS that optimizes performance of the developer's mobile app running on Windows Phone devices. Especially for Google, whose technical and business expertise rests on the processing of data, the ability to create value for users through cataloguing, sorting and serving increasingly large volumes of data may be the critical complementary asset not only for mobile, but across all sectors. Google's recent investments in both wireless and fiber optic cable to the home may coalesce into a vertical Google solution, or may just be insurance against attempts by telecommunication operators to charge exorbitant tolls for access to consumers as network neutrality rules erode. Google would own assets on the entire value chain, from operating systems and native apps to server farms, long-haul fiber, last-mile connection to the Internet, and Internet services. In this scenario, a user could live completely within the Google ecosystem, offering the company unparalleled opportunities for mining and extracting information it can monetize. While the integration strategy we posited appears to be dominant, the recent sale of the Motorola handset division to Lenovo suggests that Google no longer sees complete integration as necessary.

In an age of increasingly abundant processing power, data storage, and bandwidth, the continued growth of cloud-based services and smart edge devices seems certain. Moreover, the Internet of Things is becoming reality, with tablets, TVs, thermostats, health monitors, watches, and other "smart" devices exhibiting a range of form factors and functions, but all connecting to the Internet to find information, share data, access services, and more. The 
convergence is visible in the historically most popular networked devices - the telephone, TV, and PC - all of which are now communicating in TCP/IP. Where there once were multiple languages spoken, requiring converters to interpret and translate, now there is the potential that all things will communicate directly to each other in Internet protocols. To borrow from the Lord of the Rings, the cloud center is threatening to become the meta-platform that subsumes all of the devices. In this way, it becomes the one ring to bind and unite them all.

Acknowledgments This research is a part of the ongoing collaboration of BRIE, the Berkeley Roundtable on the International Economy at the University of California, Berkeley, and ETLA, The Research Institute on the Finnish Economy funded by Tekes. The authors thank Andrew Hargadon, Kenji Kushida, Greg Linden, Petri Rouvinen, John Zysman, and two anonymous reviewers for their helpful comments on earlier versions of the paper. We thank Casey Castaldi for her assistance on the paper.

Open Access This article is distributed under the terms of the Creative Commons Attribution License which permits any use, distribution, and reproduction in any medium, provided the original author(s) and the source are credited.

\section{References}

Abernathy WI, Utterback JM (1978) Patterns of industrial innovation. Technol Rev 80:40-47

Anderson P, Tushman ML (1990) Technological discontinuities and dominant designs: a cyclical model of technological change. Adm Sci Q 35(4):604-633

Baldwin CY, Clark KB (2000) Design rules: the power of modularity, vol 1. MIT Press, Cambridge

Boudreau K (2010) Open platform strategies and innovation: granting access vs. devolving control. Manag Sci 56(10):1849-1872

Christensen CM, Anthony SD (2004) Seeing what's next: using the theories of innovation to predict industry change. Harvard Business School Press, Boston

Chui M, Löffler M, Roberts R (2010) The internet of things. McKinsey Q 2:1-9

Cusumano M (2010) Technology strategy and management: the evolution of platform thinking. Commun ACM 53(1):32

Cusumano M, Gawer A (2001) Driving high-tech innovation: the four levers of platform leadership. Working paper 142. Center for ebusiness@, Massachusetts Institute of Technology, Cambridge

Cusumano M, Suarez F (2009) The role of services in platform markets. In: Gawer A (ed) Platforms, markets and innovation. Edward Elgar Publishing, Cheltenham

Cusumano MA, Yoffie DB (1998) Competing on internet time: lessons from netscape and its battle with microsoft. The Free Press, New York

Eisenmann T, Parker G, Van Alstyne M (2009) Opening platforms: how, when and why? Working Paper 09-030. Harvard Business School, Boston

Eisenmann T, Parker G, Van Alstyne M (2011) Platform envelopment. Strateg Manag J 32(12):1270-1285

Farrell J, Saloner G (1992) Converters, compatibility, and the control of interfaces. J Ind Econ 40(1):9-35

Gawer A (2011) Platforms, markets and innovation. Edward Elgar Publishing, Cheltenham

Gawer A, Cusumano MA (2002) Platform leadership. Harvard Business School Press, Boston

Gerschenfeld N, Krikorian R, Cohen D (2004) The internet of things. Sci Am 291(4):76-81

Jacobides MG, Knudsen T, Augier M (2006) Benefiting from innovation: value creation, value appropriation and the role of industry architectures. Res Policy 35(8):1200-1221

Katz ML, Shapiro C (1985) Network externalities, competition, and compatibility. Am Econ Rev 75(3):424-440

Kenney M, Curry J (2002) The Internet and the personal computer value chain. In: BRIE-IGCC E-conomy Project (ed) Tracking a transformation. Brookings Institution Press, Washington DC, pp 151-177

Kenney M, Pon B (2011) Structuring the smartphone industry: is the mobile internet OS platform the key? J Ind Compet Trade 11(3):239-261

Klein B (2001) The Microsoft case: what can a dominant firm do to defend its market position? J Econ Perspect $15(2): 45-62$ 
Kushida KE, Murray J, Zysman J (2011) Diffusing the cloud: cloud computing and implications for public policy. J Ind Compet Trade 11(3):209-237

Murray J (2013) Economics and platform architecture. Blog post. http://www.adamalthus.com/blog/2013/02/27/ economics-and-platform-architecture-i/. Accessed 18 April 2013

Pon B, Seppala T, Kenney M (2014) Android and the demise of operating system-based power: firm strategy and platform control in the post-PC world. Telecommun Policy 38:979-991

Rochet JC, Tirole J (2003) Platform competition in two-sided markets. J Eur Econ Assoc 1(4):990-1029

Schilling MA (2009) Protecting or diffusing a technology platform: tradeoffs in appropriability, network externalities, and architectural control. In: Gawer A (ed) Platforms, markets and innovation. Edward Elgar Publishing, Cheltenham, pp 192-218

Seppälä T, Broens R (2013) How new smartwatches could revolutionize the artefact surroundings of people. ETLA Brief No. 5. The Research Institute of the Finnish Economy, Helsinki

Tee R, Gawer A (2009) Industry architecture as a determinant of successful platform strategies: a case study of the i-mode mobile Internet service. Eur Manag Rev 6(4):217-232

Teece DJ (1986) Profiting from technological innovation: implications for integration, collaboration, licensing and public policy. Res Policy 15:285-305

West J (2003) How open is open enough? Melding proprietary and open source platform strategies. Res Policy 32(7):1259-1285

West J, Mace M (2010) Browsing as the killer app: explaining the rapid success of Apple's iPhone. Telecommun Policy 34(5):270-286 